\title{
Short-Term Ventricular Assist Devices (Implantable and Percutaneous)
}

\author{
Hiroo Takayama $\cdot$ Lauren Truby $\cdot$ Koji Takeda \\ Yoshifumi Naka
}

Published online: 29 April 2014

(C) Springer Science + Business Media New York 2014

\begin{abstract}
Short-term ventricular assist devices (VADs), percutaneous or surgical, are used in the settings of cardiogenic shock (CS) refractory to medical management, as bridges to myocardial recovery, heart transplantation, durable long-term devices, and in the aid of high-risk PCIs. Recent innovations in continuous-flow pump technology have contributed to the development of various short-term VADs with many impressive features. These include the CentriMag VAD and percutaneous VADs such as the Impella and TandemHeart VAD systems. With these and other advances in mechanical circulatory support, it is highly likely that the significant morbidity and mortality associated with CS are improving. However, clinical evidence supporting the use of these devices remains at the level of small case series. Rigorous clinical investigation is warranted to better quantify the contributions of device therapy to the management of CS.
\end{abstract}

Keywords Cardiogenic shock $\cdot$ Short-term ventricular assist devices $\cdot$ Implantable $\cdot$ Percutaneous $\cdot$ Short-term vent $\cdot$ CentriMag $\cdot$ Impella $\cdot$ TandemHeart

This article is part of the Topical Collection on Artificial Organ CT Surgery.

H. Takayama $(\bowtie) \cdot$ L. Truby $\cdot$ K. Takeda $\cdot$ Y. Naka

Department of Surgery, Columbia University Medical Center,

177 Fort Washington Ave, MHB 7-435, New York, NY 10032,

USA

e-mail: ht2225@cumc.columbia.edu

L. Truby

e-mail: 1kt2118@cumc.columbia.edu

K. Takeda

e-mail: kt2485@cumc.columbia.edu

Y. Naka

e-mail: yn33@cumc.columbia.edu

\section{Introduction}

Advances in mechanical circulatory support device (MCSD) technology have led to widespread application of ventricular assist devices (VADs) in various forms of heart failure. In particular, the application of the durable continuous-flow left ventricular assist device (CF-LVAD) in the advanced stages of chronic heart failure has become the standard of care [1]. The technology associated with durable LVADs continues to evolve, and it is likely that we will soon see its relevance in the earlier stages of heart failure. However, current evidence suggests that this therapy, in its current form, does not provide satisfactory outcomes in those patients with more acute forms of disease (e.g., cardiogenic shock or INTERMACS I patients) [2, 3]. An INTERMACS 1 profile has repeatedly been identified as a risk factor for mortality in previous studies of durable LVAD technology [4]. Unsatisfactory outcomes, the emergent nature of INTERMACS I cardiogenic shock (CS), and the need for biventricular support may not justify the costly and aggressive use of durable LVAD technology for short-term MCS. It is in this patient population that the 'shortterm VAD' plays an important role [5]. These devices can also be used in patients whose myocardial function is expected to recover in a short period of support time, such as postcardiotomy shock or graft dysfunction after heart transplantation. The purpose of this review is to provide a comprehensive update on the current status of short-term VADs, to illustrate their clinical application in our program, and finally to offer a perspective on the future direction of these technologies.

\section{What is 'Short-Term Vad'?}

There is no clear definition of 'short-term VAD.' Although the term is often used in the context of extracorporeal 
MCS, some devices are designed as 'paracorporeal.' INTERMACS defines 'durable MCS' as those devices that feasibly permit discharge from the hospital. Therefore, for the purposes of this review, we will define 'short-term MCSD' as MCSDs used only in the in-patient treatment setting. Utilizing this definition, paracorporeal VADs, such as the PVAD (Thoratec Corp., Pleasanton, CA), are considered a durable MCSD and will not be discussed here. Short-term MCSDs can be further divided into two main subtypes: the VA ECMO and short-term VADs. This article will only focus on the short-term VADs, inclusive of both methods of insertion: percutaneous and surgical.

\section{Percutaneous Short-Term VAD}

The most important recent update on percutaneous MCS was the IABP-SHOCK II trial, which failed to show a survival benefit of intraaortic balloon pump (IABP) therapy when compared to medical management in the setting of acute myocardial infarction (AMI)/CS [6•]. Another important message from this study was the persistently high 30-day mortality of $\sim 40 \%$ in this cohort of patients. Expert opinion suggests that the study of novel and innovative devices and therapies is warranted, and trials of percutaneous VADs (pVADs) or VA ECMO are likely to be the next on the horizon [7]. Percutaneous MCS is advantageous in the setting of acute CS, where it is less invasive for unstable patients and decreases morbidity associated with surgical placement of MCSD. However, it has its own limitations, such as flow capacity, support period (days), need for bed rest, as well as need for an appropriate placement setting (catheterization laboratory, except VA ECMO). Current studies of available devices have failed to show a survival benefit despite obvious hemodynamic improvement. In the meta-analysis of three studies comparing pVAD (two with TandemHeart and one with Impella 2.5) to IABP, pVAD did not improve 30-day mortality (RR 1.06, $95 \%$ CI 0.68-1.66) [8]. With the introduction of newer devices and technology, however, pVADs will likely have a significant impact on the field in the near future. The following discussion will review each type of percutaneous and surgical VAD, as well as review the Columbia University algorithm for MCSD evaluation and management.

Impella LVAD System (ABIOMED, Inc., Pittsburgh, PA)

The Impella LVAD system is a catheter-mounted CF axial pump placed across the aortic valve (Fig. 1). The inflow is placed in the LV and the outflow in the aorta. There are several versions of the Impella: $2.5(12 \mathrm{~F}, 2.5 \mathrm{l} / \mathrm{min}$ of flow), CP (14 F, 3.5 1/min of flow), 5.0 (21 F, 5 1/min of flow), and $\operatorname{LD}(21 \mathrm{~F}, 5 \mathrm{l} / \mathrm{min}$ of flow). Another subtype for $\mathrm{RV}$ support is in the early phases of clinical use [9]. The insertion procedure is relatively straightforward under fluoroscopic (and echocardiographic) guidance and allows

Fig. 1 Impella LVAD system. (Courtesy of ABIOMED, Inc.)

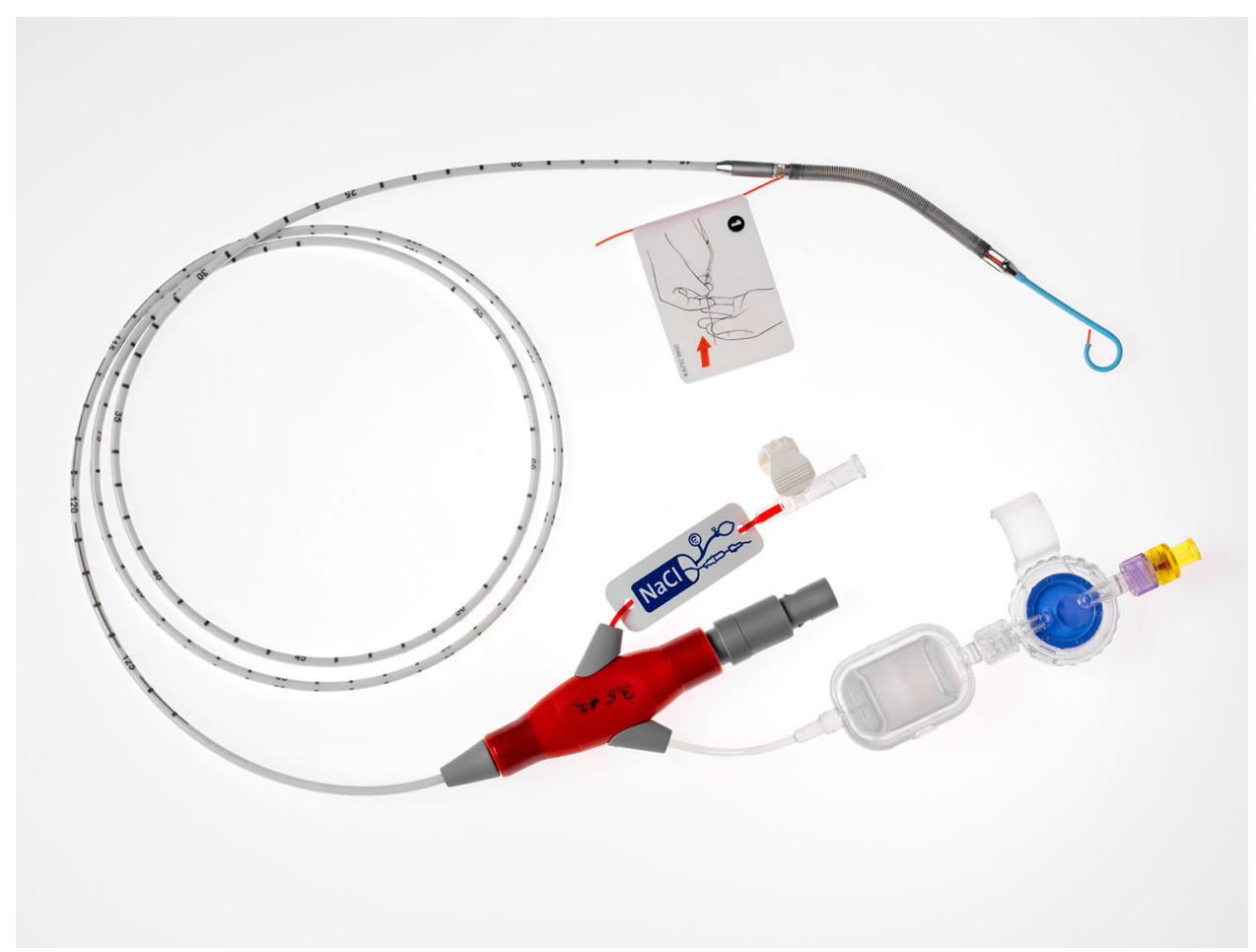


for prompt initiation of support. The system provides partial decompression of the ventricle, which might contribute to myocardial unloading and ultimate recovery. Here, we will discuss the 2.5 and $\mathrm{CP}$, as the 5.0 and $\mathrm{LD}$ require surgical cut-down and are described later in the article.

Impella 2.5 is the most widely used subtype and is particularly useful to aid complex PCIs. Because of the ease of insertion in the catheterization laboratory, this device has also been applied to CS in the setting of AMI or PCI complications. When compared to the IABP, Impella 2.5 LVAD provided superior hemodynamic support and was both feasible and safe [10]. However, Impella 2.5 LVAD failed to provide a 30-day mortality benefit in this randomized study. These results were recently reproduced in a retrospective analysis of 120 patients who were enrolled in the multicenter Impella-EUROSHOCK Registry [11]. A maximal flow of $2.5 \mathrm{l} / \mathrm{min}$ appears to limit its clinical benefit in patients with profound CS. One unique application of Impella 2.5 is its use in combination with VA ECMO to facilitate LV decompression [12]. With a maximal flow of $3.5 \mathrm{l} / \mathrm{min}$, Impella $\mathrm{CP}$, which has recently become clinically available in the US, might overcome the flow limitations of Impella 2.5. The Impella CP pump has a slightly larger diameter yet can still be inserted percutaneously, preserving the ease of insertion.

The TandemHeart pVAD (CardiacAssist, Inc., Pittsburgh, PA)

The TandemHeart is a continuous-flow pump that can generate up to $4 \mathrm{l} / \mathrm{min}$ of flow at 7,500 rpm and can be inserted percutaneously in the cardiac catheterization laboratory. To establish LV hemodynamic support, a 21-F inflow cannula is inserted from the femoral vein into the left atrium through a trans-septal puncture, and a 15- or 17-F outflow cannula is inserted into the femoral artery. The pump can also serve as an RVAD when connected to cannulas positioned in the pulmonary artery and the right atrium.

In one study, patients with AMI/CS were randomized to either IABP $(n=20)$ or TandemHeart LVAD $(n=21)$ [13]. The cardiac power index, as well as other hemodynamic and metabolic variables, improved more substantially with TandemHeart LVAD support. However, complications such as severe bleeding and limb ischemia were more frequent with the pVAD. Thirty-day mortality was not significantly different (IABP $45 \%$ vs. pVAD $43 \%$ ). More recently, the excellent clinical potential of this device was reported in a single institution case series by Kar et al. [14•]. A total of 117 patients with refractory CS were supported with this device. Forty-eight percent $(n=56)$ were undergoing active cardiopulmonary resuscitation immediately before or at the time of insertion. Eighty patients had ischemic and 37 patients had nonischemic cardiomyopathy. The average duration of support was $5.8 \pm 4.75$ days. After implantation, the cardiac index, systolic blood pressure, mixed venous oxygen saturation, urine output, pulmonary capillary wedge pressure, lactic acid level, and creatinine level all improved. The 30-day and 6-month mortality rates were 40.2 and $45.3 \%$, respectively. Although additional small case series have reported successful application of this device in various forms of CS [15-17], widespread use is limited. This is likely due to the technical complexity of trans-septal puncture in the emergent/urgent situation as well as the unclear clinical benefit. Ostadal et al. [18] examined the hemodynamic effect of three types of percutaneous MCS in a porcine model: VA ECMO, TandemHeart LVAD, and Impella 2.5 LVAD. Under ventricular fibrillation, VA ECMO was the most efficacious in maintaining mean arterial pressure, followed by the TandemHeart LVAD and the Impella 2.5 LVAD.

\section{Surgical Short-Term Vad}

Surgical short-term VADs enable generation of sufficient flow and demonstrate increased durability as compared to pVADs. However, they do require surgical cut-down and often sternotomy. The history of surgical VADs begins with the development of the pulsatile flow pump. The Abiomed BVS5000 (ABIOMED Inc.) was the first FDAapproved extracorporeal pulsatile pneumatic device. The next generation was 'paracorporeal' pulsatile VADs, such as the PVAD (Thoratec Corp., Pleasanton, CA) and Abiomed AB5000 (ABIOMED Inc.), with which patients could be discharged.

\section{CentriMag VAD (Thoratec Corp., Pleasanton, CA)}

CentriMag VAD is the most recently introduced short-term VAD (Fig. 2). Based on CF technology, the CentriMag utilizes a magnetic levitation bearingless centrifugal pump, which can generate up to $10 \mathrm{l} / \mathrm{min}$ of flow at $5,500 \mathrm{rpm}$. This pump has rapidly become the surgical short-term VAD of choice in CS at our institution as well as other centers. CentriMag VAD facilitates an easy insertion procedure. It allows for flexible configuration, combined use with oxygenator, and long-term use. In addition, its postoperative maintenance allows ambulatory rehabilitation [19]. The device is ideal for bridge-to-decision therapy. Since Mueller et al. [20] reported their initial experience on 11 patients with the CentriMag VAD in 2004, a number of small case series have been published from around the world [21-24]. In a multicenter study of 38 patients with refractory CS, CentriMag devices were implanted in a left $(n=8)$, right $(n=12)$, or biventricular $(n=18)$ configuration [25]. The mean 

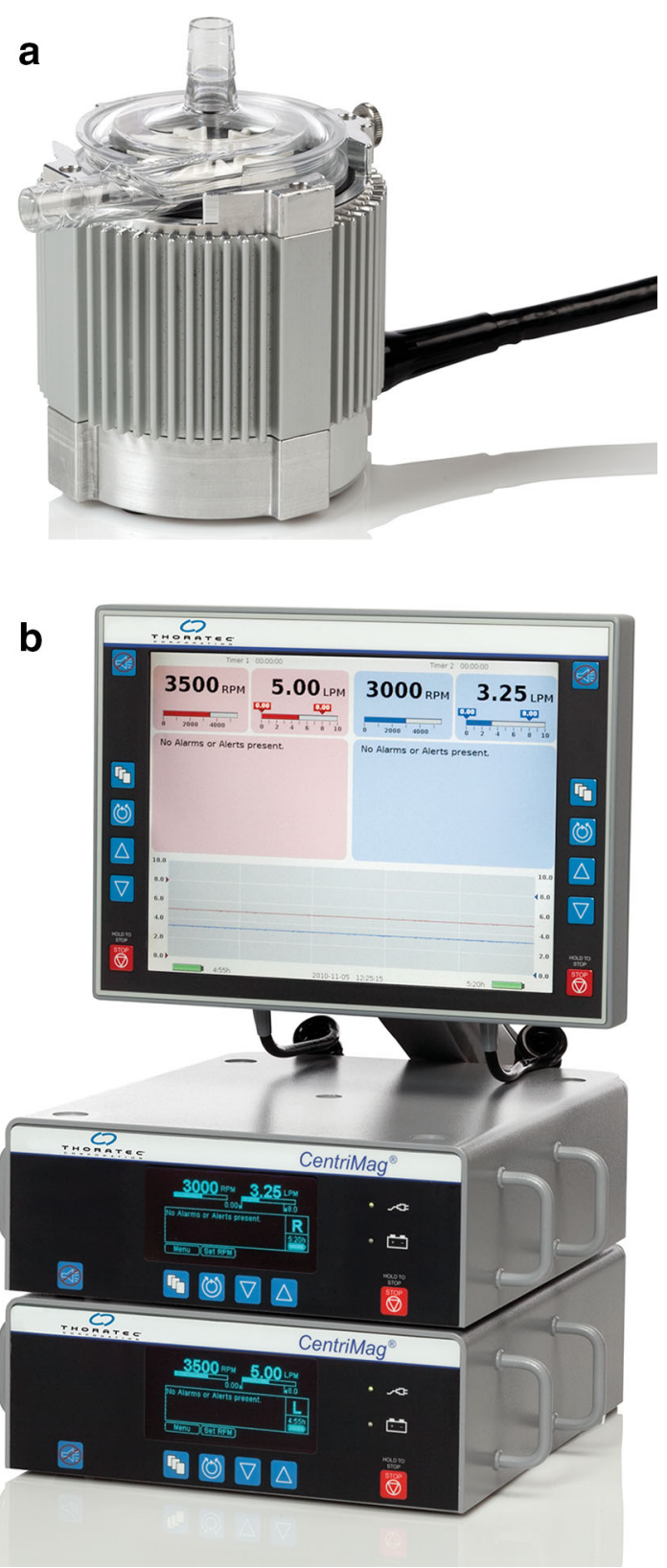

Fig. 2 a CentriMag pump. (Courtesy of Thoratec Corp.) b Console of the CentriMag VAD system. (Courtesy of Thoratec Corp.)

duration of support was 13 days (1-60 days), and survival at 30 days was $47 \%$. Mohite et. al. [26] reported outcomes from extended use (beyond 30 days) in 41 patients. Unique insertion techniques have also been reported, including a minithoracotomy and a percutaneous approach [27, 28]. Of note, compatibility of the system to different cannulas allows for connection to other types of VADs, such as the Berlin Heart Excor and PVAD [29, 30]. CentriMag VAD use has also expanded to the pediatric population [31] and to the ECMO setting [21]. In our program, over 150 patients received CentriMag VAD for various indications such as CS refractory to medical management, postcardiotomy shock, graft failure post-heart transplantation, and RV failure post-implantable LVAD. BiVAD was used in over $60 \%$ of cases in order to achieve supra-normal cardiac indices. Heparin was used for anticoagulation. The longest support period was 145 days. Major bleeding occurred in $1 / 3$ of the patients and cerebrovascular accident in over $10 \%$. There was no CentriMag pump failure or thrombosis in our case series.

Impella 5.0 and LD (ABIOMED Inc.)

Impella 5.0 requires a hybrid approach for insertion. Because of its pump size ( $21 \mathrm{~F})$, a peripheral artery, either the femoral artery or axillary artery, is exposed through a surgical cutdown, and the pump is inserted and positioned under fluoroscopic (and echocardiographic) guidance. Axillary artery access requires anastomosis of a chimney graft, whereas femoral artery access allows direct insertion through a purse string suture. Axillary insertion allows ambulatory rehabilitation. The Impella LD is inserted through the ascending aorta, approached through a thoracotomy or a sternotomy. Both devices provide up to $5 \mathrm{l} / \mathrm{min}$ of flow. Impella 5.0 has been shown in single cases to be effective as a bridge-totransplantation [32], bridge-to-bridge [33], and bridge-torecovery device [34]. Lemaire et al. [35] reported 47 patients who received Impella support, $80 \%$ of whom received either the Impella 5.0 or LD. Indications included CS refractory to medical management in 15 patients $(32 \%)$ and postcardiotomy shock in $32(68 \%)$. The mean duration of support was $5.4 \pm 4.5$ days (range: 1-18 days). Ventricular function recovered in 34 of 47 patients $(72 \%)$ resulting in device removal. The 30-day mortality was $25 \%$ (12 of 47 patients). Complications, such as device malfunction, high purge pressures, tube fracture, and groin hematoma, occurred in 14 patients $(30 \%)$

\section{Columbia Strategy for Use of the Short-Term VAD}

At our center, when a patient who remains in CS despite maximal medical management is identified, the patient is rapidly evaluated for MCSD placement (Fig. 3) [36•]. Early recognition of refractory shock through physical examination (cool extremities, tachycardia, decreasing urine output), rather than waiting for laboratory evidence of end-organ failure, allows for initiation of MCS in a timely fashion. Once the decision has been made to provide mechanical support, a short-term MCSD (CentriMag VAD or VA ECMO) is promptly placed. As of 2013 , neither Impella pVAD nor TandemHeart pVAD is the device of choice in CS in our program because of limitations in flow, duration of support, and need for catheterization laboratory availability for insertion. The surgical CentriMag VAD is used whenever feasible. However, VA ECMO is chosen in conditions such as unclear 
Fig. 3 Treatment algorithm for cardiogenic shock

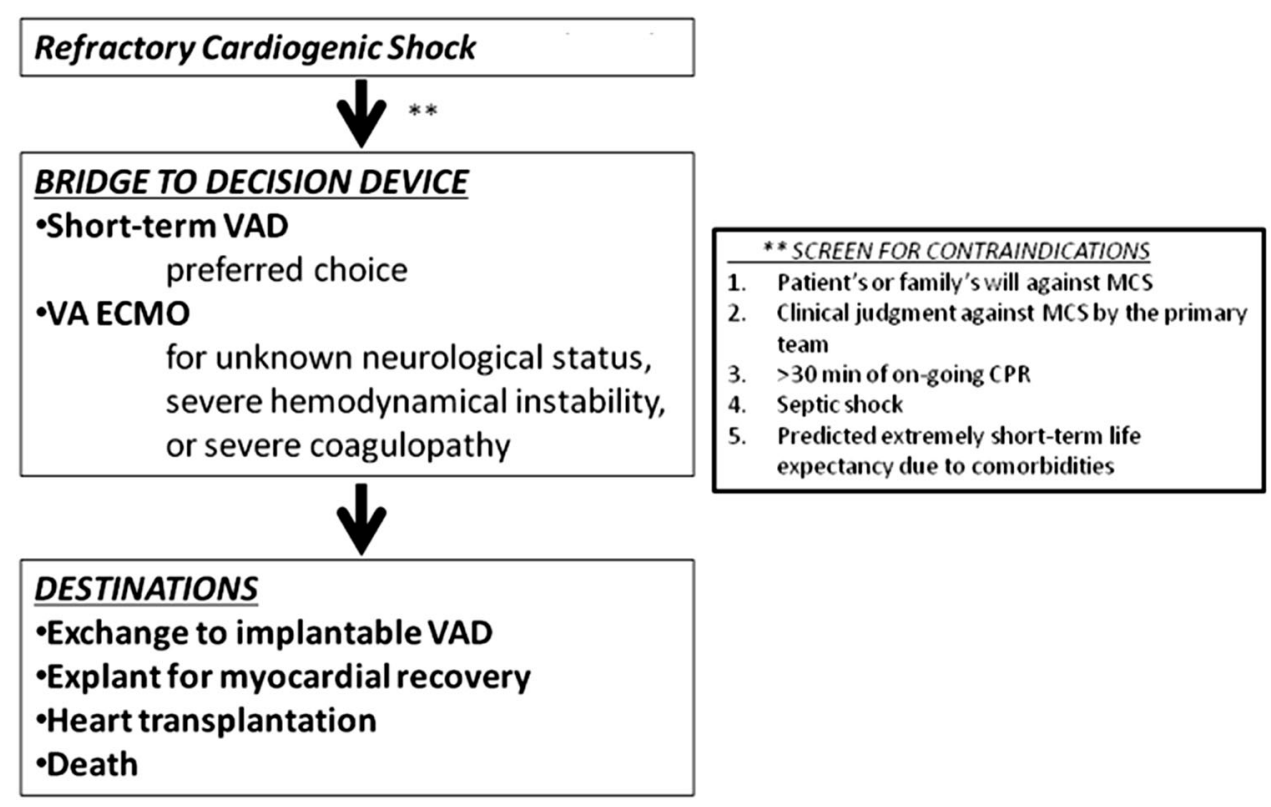

neurological status, unstable hemodynamics, and severe coagulopathy. After resuscitation of the patient with the shortterm MCSD, the patient's myocardial function is evaluated by weaning from the device under echocardiographic and hemodynamic monitoring. Depending on the result of this weaning trial and eligibility for heart replacement therapy, the short-term MCSD is explanted as a transition to one of the following destinations: exchange to durable MCSD, explantation for myocardial recovery, or heart transplantation. The multidisciplinary MCSD team is involved in all stages of patient care: evaluation of CS patients, management of patients on short-term MCSD, performing weaning studies, and the evaluation for heart transplantation or implantable LVAD for destination therapy.

From January 2007 through January 2012, 90 patients received MCSD through this algorithm for CS, with $23 \%$ receiving active cardiopulmonary resuscitation at the time of device placement [36॰]. Those patients with postcardiotomy shock and graft failure post-heart transplantation were not included. Etiologies of CS included AMI in $49 \%$ and acute decompensated heart failure in $27 \%$. CentriMag VAD was used in $49 \%$ and VA ECMO in $51 \%$. Median length of support was 8 days (IQR, 4-18 days). Exchange to implantable VAD was required in $26 \%$. Other destinations included myocardial recovery in $18 \%$ and heart transplantation in $11 \%$. Survival to hospital discharge was $49 \%$.

\section{Future Direction}

The mortality of patients who develop CS remains high, ranging from 50 to $80 \%[37,38]$. Improvement of this persistently and unacceptably high mortality requires significant breakthroughs in multiple facets of CS management, not limited to device technology.

Although multiple devices are now available to meet the specific needs of each CS patient, the decision-making surrounding device therapy often complicates patient care. First, to streamline the management of CS, we suggest a multidisciplinary MCSD team, consisting of cardiothoracic surgeons, heart failure cardiologists, intensive care specialists, nurse practitioners, physical therapists, and dieticians. In this way, physicians and healthcare providers familiar with the unique challenges posed by these new technologies directly manage CS patients who require device therapy.

Second, demonstration of clinical efficacy and noninferiority of device therapy has to be achieved for integration of MCS into the mainstream treatment of CS. This requires well-designed clinical investigation. In order to properly design studies, however, a clear definition of refractory CS must be established by vigorous data review and consensus agreement.

Lastly, further improvements in device therapy algorithms are warranted. These include identification of appropriate timing for MCS therapy and further improvement in device technology.

\section{Conclusions}

Recent innovations in CF technology have contributed to the development of various short-term VADs with impressive features, and as a result, it is likely that 
outcomes of CS are improving. pVADs are becoming smaller and more powerful. Surgical extracorporeal CF short-term VADs are becoming more popular; and as the next generation of surgical durable VADs emerges, they could easily become more integrated into CS management. Clinical evidence for each device, however, remains at the level of case reports. Equally important to the evolution of technology is the pursuit of robust clinical investigations to quantify the contribution of device therapy to CS.

\section{Compliance with Ethics Guidelines}

Conflict of Interest Hiroo Takayama, Lauren Truby, Koji Takeda, and Yoshifumi Naka declare that they have no conflict of interest.

Human and Animal Rights and Informed Consent This article does not contain any studies with animal subjects performed by any of the authors. With regard to the authors' research cited in this paper, all procedures were followed in accordance with the ethical standards of the responsible committee on human experimentation and with the Helsinki Declaration of 1975, as revised in 2000 and 2008.

\section{References}

Papers of particular interest, published recently, have beenhighlighted as:

- Of importance

1. Peura JL, Colvin-Adams M, Francis GS, et al. Recommendations for the use of mechanical circulatory support: device strategies and patient selection: a scientific statement from the American Heart Association. Circulation. 2012;126:2648-67.

2. Boyle AJ, Ascheim DD, Russo MJ, et al. Clinical outcomes for continuous-flow left ventricular assist device patients stratified by pre-operative INTERMACS classification. J Heart Lung Transplant. 2011;30:402-7.

3. Stevenson LW, Pagani FD, Young JB, et al. INTERMACS profiles of advanced heart failure: the current picture. J Heart Lung Transplant. 2009;28:535-41.

4. Kirklin JK, Naftel DC, Kormos RL, et al. Fifth INTERMACS annual report: risk factor analysis from more than 6,000 mechanical circulatory support patients. J Heart Lung Transplant. 2013;32:141-56.

5. Worku B, Naka Y, Pak SW, et al. Predictors of mortality after short-term ventricular assist device placement. Ann Thorac Surg. 2011;92:1608-12 (discussion 1612-3).

6. - Thiele H, Zeymer U, Neumann FJ et al. Intraaortic balloon support for myocardial infarction with cardiogenic shock. New Engl J Med. 2012;367:1287-96. This randomized study questioned what the standard of care is for IABP use in AMI/CS. Also importantly the study showed persistently high mortality similar to the original SHOCK trial, suggesting the importance of implementation of MCSD.

7. O'Connor CM, Rogers JG. Evidence for overturning the guidelines in cardiogenic shock. New Engl J Med. 2012;367:1349-50.

8. Cheng JM, den Uil CA, Hoeks SE, et al. Percutaneous left ventricular assist devices vs. intra-aortic balloon pump counterpulsation for treatment of cardiogenic shock: a meta-analysis of controlled trials. Eur Heart J. 2009;30:2102-8.
9. Margey R, Chamakura S, Siddiqi S, et al. First experience with implantation of a percutaneous right ventricular Impella right side percutaneous support device as a bridge to recovery in acute right ventricular infarction complicated by cardiogenic shock in the United States. Circ Cardiovasc Interv. 2013;6:e37-8.

10. Seyfarth M, Sibbing D, Bauer I, et al. A randomized clinical trial to evaluate the safety and efficacy of a percutaneous left ventricular assist device versus intra-aortic balloon pumping for treatment of cardiogenic shock caused by myocardial infarction. J Am Coll Cardiol. 2008;52:1584-8.

11. Lauten A, Engstrom AE, Jung C, et al. Response to letter regarding article, "percutaneous left-ventricular support with the impella-2.5-assist device in acute cardiogenic shock results of the Impella-EUROSHOCK registry". Circ Heart Fail. 2013;6:e56.

12. Koeckert MS, Jorde UP, Naka Y, Moses JW, Takayama H, Impella LP. 2.5 for left ventricular unloading during venoarterial extracorporeal membrane oxygenation support. J Card Surg. 2011;26:666-8.

13. Thiele H, Sick P, Boudriot E, et al. Randomized comparison of intra-aortic balloon support with a percutaneous left ventricular assist device in patients with revascularized acute myocardial infarction complicated by cardiogenic shock. Eur Heart J. 2005;26:1276-83.

14. - Kar B, Gregoric ID, Basra SS, Idelchik GM, Loyalka P. The percutaneous ventricular assist device in severe refractory cardiogenic shock. J Am Coll Cardiol. 2011;57:688-96. This singlecenter case series reports excellent outcomes using pVAD, TandemHeart VAD system.

15. Brinkman WT, Rosenthal JE, Eichhorn E, et al. Role of a percutaneous ventricular assist device in decision making for a cardiac transplant program. Ann Thorac Surg. 2009;88:1462-6.

16. Giesler GM, Gomez JS, Letsou G, Vooletich M, Smalling RW. Initial report of percutaneous right ventricular assist for right ventricular shock secondary to right ventricular infarction. Catheter Cardiovasc Interv. 2006;68:263-6.

17. Takagaki M, Wurzer C, Wade R, et al. Successful conversion of TandemHeart left ventricular assist device to right ventricular assist device after implantation of a HeartMate XVE. Ann Thorac Surg. 2008;86:1677-9.

18. Ostadal P, Mlcek M, Holy F, et al. Direct comparison of percutaneous circulatory support systems in specific hemodynamic conditions in a porcine model. Circ Arrhythm Electrophysiol. 2012;5:1202-6.

19. Takayama H, Chen JM, Jorde UP, Naka Y. Implantation technique of the CentriMag biventricular assist device allowing ambulatory rehabilitation. Interact Cardiovasc Thorac Surg. 2011;12:110-1.

20. Mueller JP, Kuenzli A, Reuthebuch O, et al. The CentriMag: a new optimized centrifugal blood pump with levitating impeller. Heart Surg Forum. 2004;7:E477-80.

21. Aziz TA, Singh G, Popjes E, et al. Initial experience with CentriMag extracorporal membrane oxygenation for support of critically ill patients with refractory cardiogenic shock. J Heart Lung Transplant. 2010;29:66-71.

22. De Robertis F, Rogers P, Amrani M, et al. Bridge to decision using the Levitronix CentriMag short-term ventricular assist device. J Heart Lung Transplant. 2008;27:474-8.

23. Worku B, Naka Y, Pak SW, et al. Predictors of mortality after short-term ventricular assist device placement. Ann Thorac Surg. 2011;92:1608-12 (discussion 1612-3).

24. Loforte A, Montalto A, Ranocchi F, et al. Levitronix CentriMag third-generation magnetically levitated continuous flow pump as bridge to solution. ASAIO J. 2011;57:247-53.

25. John R, Long JW, Massey HT, et al. Outcomes of a multicenter trial of the Levitronix CentriMag ventricular assist system for short-term circulatory support. J Thorac Cardiovasc Surg. 2011;141:932-9. 
26. Mohite PN, Zych B, Popov AF, et al. CentriMag(R) short-term ventricular assist as a bridge to solution in patients with advanced heart failure: use beyond 30 days. Eur J Cardiothorac Surg. 2013;44:e310-5.

27. Takayama H, Naka Y, Jorde UP, Stewart AS. Less invasive left ventricular assist device placement for difficult resternotomy. J Thorac Cardiovasc Surg. 2010;140:932-3.

28. Takayama H, Naka Y, Kodali SK, et al. A novel approach to percutaneous right-ventricular mechanical support. Eur J Cardiothorac Surg. 2012;41:423-6.

29. Maat AP, van Thiel RJ, Dalinghaus M, Bogers AJ. Connecting the Centrimag Levitronix pump to Berlin Heart Excor cannulae; a new approach to bridge to bridge. J Heart Lung Transplant. 2008;27:112-5.

30. Jaroszewski DE, Marranca MC, Pierce CN, et al. Successive circulatory support stages: a triple bridge to recovery from fulminant myocarditis. J Heart Lung Transplant. 2009;28:984-6.

31. Hirata Y, Charette K, Mosca RS, Quaegebeur JM, Chen JM. Pediatric application of the Thoratec CentriMag BiVAD as a bridge to heart transplantation. J Thorac Cardiovasc Surg. 2008;136:1386-7.

32. LaRocca GM, Shimbo D, Rodriguez CJ, et al. The Impella Recover LP 5.0 left ventricular assist device: a bridge to coronary artery bypass grafting and cardiac transplantation. J Am Soc Echocardiogr. 2006;19:468.e5-7.

33. Samoukovic G, Rosu C, Giannetti N, Cecere R. The Impella LP 5.0 as a bridge to long-term circulatory support. Interact Cardiovasc Thorac Surg. 2009;8:682-3.

34. Andrade JG, Al-Saloos H, Jeewa A, Sandor GG, Cheung A. Facilitated cardiac recovery in fulminant myocarditis: pediatric use of the Impella LP 5.0 pump. J Heart Lung Transplant. 2010;29:96-7.

35. Lemaire A, Anderson MB, Lee LY, et al. The impella device for acute mechanical circulatory support in patients in cardiogenic shock. Ann Thorac Surg. 2013;97(1):133-8.

36. - Takayama H, Truby L, Koekort M et al. Clinical outcome of mechanical circulatory support for refractory cardiogenic shock in the current era. J Heart Lung Transplant. 2013;32:106-11. This single-center case series provides an overview of the current status of device therapy for CS various settings.

37. Goldberg RJ, Samad NA, Yarzebski J, Gurwitz J, Bigelow C, Gore JM. Temporal trends in cardiogenic shock complicating acute myocardial infarction. N Engl J Med. 1999;340:1162-8.

38. Holmes DR Jr, Berger PB, Hochman JS, et al. Cardiogenic shock in patients with acute ischemic syndromes with and without STsegment elevation. Circulation. 1999;100:2067-73. 